\title{
Effects of multiple scattering on angle-independent structural color in disordered colloidal materials
}

\author{
Victoria Hwang, ${ }^{1, *}$ Anna B. Stephenson, ${ }^{1}, *$ Sofia Magkiriadou, $,{ }^{2}, \dagger$ Jin-Gyu Park, ${ }^{1,}{ }^{\ddagger}$ and Vinothan N. Manoharan ${ }^{1,2, \S}$ \\ ${ }^{1}$ Harvard John A. Paulson School of Engineering and Applied Sciences, \\ Harvard University, 29 Oxford Street, Cambridge, Massachusetts 02138, USA \\ ${ }^{2}$ Department of Physics, Harvard University, 17 Oxford Street, Cambridge, Massachusetts 02138, USA
}

(Dated: July 15, 2019)

\begin{abstract}
Disordered packings of colloidal spheres show angle-independent structural color when the particles are on the scale of the wavelength of visible light. Previous work has shown that the positions of the peaks in the reflectance spectra can be predicted accurately from a single-scattering model that accounts for the effective refractive index of the material. This agreement shows that the main color peak arises from short-range correlations between particles. However, the single-scattering model does not quantitatively reproduce the observed color: the main peak in the reflectance spectrum is much broader and the reflectance at low wavelengths is much larger than predicted by the model. We use a combination of experiment and theory to understand these features. We find that one significant contribution to the breadth of the main peak is light that is scattered, totally internally reflected from the boundary of the sample, and then scattered again. The high reflectance at low wavelengths also results from multiple scattering but can be traced to the increase in the scattering cross-section of individual particles with decreasing wavelength. Both of these effects tend to reduce the saturation of the structural color, which limits the use of these materials in applications. We show that while the single-scattering model cannot reproduce the observed saturations, it can be used to design materials in which multiple scattering is suppressed and the color saturated, even in the absence of absorbing components.
\end{abstract}

DOI:

\section{INTRODUCTION}

Structural color comes from constructive interference between waves scattered from a material with refractiveindex variations at the scale of visible light. When the index variation is periodic, as in photonic crystals [1], the structural color is angle-dependent or iridescent. But when the index variation has only short-range order, the structural color is independent of angle. Angleindependent structural colors appear matte and homogeneous, often indistinguishable from colors that come from absorbing pigments. This type of coloration is found in many species of birds [2-9] and has been mimicked in disordered assemblies of colloidal particles [10-29].

To explain and predict angle-independent structural color in these colloidal systems, Magkiriadou and colleagues [20] developed a model based on a singlescattering approximation and effective-medium theory. The model, which assumes that the particles are packed into a glassy arrangement (Fig. 1a), predicts that the primary peak in the reflectance spectrum is determined by the peak of the structure factor, which accounts for constructive interference arising from the short-range corre-

\footnotetext{
* These authors contributed equally to this work.

$\dagger$ Present address: Laboratory of Experimental Biophysics, Institute of Physics, École Polytechnique Fédérale de Lausanne, CH1015 Lausanne, Switzerland.

¥ Present address: E Ink Corporation, 1000 Technology Park Drive, Billerica, MA 01821, USA.

$\S$ vnm@seas.harvard.edu
}

lations between particles. The model also predicts that the reflectance spectrum should include contributions from the form factor, which accounts for the wavelengthdependent scattering from individual particles. The positions of the peaks predicted for both the structure and form factors agree well with those observed in experiment. In comparison to numerical methods for predicting the color, such as finite-difference time domain and finite-element methods [8,30-35], the single-scattering model gives physical insight into the reflectance peak and how it varies with the particle size, packing density, and refractive index.

However, the single-scattering model does not reproduce other features of the observed reflectance spectra: it underestimates the reflectance at short wavelengths and the breadth of the primary peak (Fig. 1b). These features likely arise from multiple scattering. Evidence for multiple scattering comes from studies showing that adding absorbers and reducing the sample thickness generally increases the saturation of the structural color $[15,17,18,27,28,36]$. Also, Noh and colleagues confirmed that multiple scattering is present in structural colors produced by sphere-type disordered structures in the barbs of bird feathers [6].

Through a combination of experiment and theory, we explain several multiple scattering effects in the reflectance spectra of disordered packings of spherical particles. We perform polarization experiments and reflectance measurements to show that a secondary peak from multiple scattering explains the breadth of the main color peak. We also use single-scattering theory to understand the onset of multiple scattering and its increase 

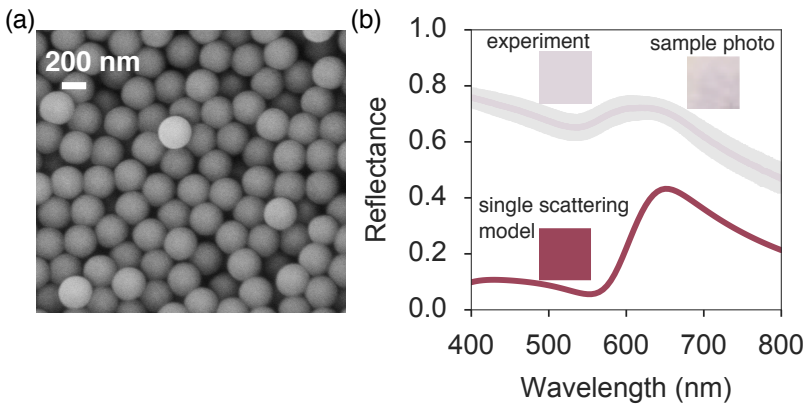

FIG. 1. (a) Scanning electron micrograph of an angleindependent structurally colored film made from $280 \mathrm{~nm}$ polystyrene spheres. (b) Experimental reflectance spectrum (light pink line) and predictions of single-scattering model (dark red line) for a disordered film of $280 \mathrm{~nm}$ polystyrene spheres and an effective refractive index ranging from 1.322 at $400 \mathrm{~nm}$ to 1.296 at $800 \mathrm{~nm}$. Reflectance is measured from all scattering angles with an integrating sphere. Line colors are calculated from spectra as described in Section II. Error bars are shown in grey for each data point and are twice the standard deviation of 6 measurements from different areas of the sample film. Insets above lines are color swatches of the calculated colors. Inset in top right is a photograph of the sample.

at short wavelengths. We validate this physical picture by developing a design rule that allows one to reduce the amount of multiple scattering and hence increase the color saturation.

The effects of multiple scattering on backscattering from disordered colloidal samples have been studied extensively in other contexts, such as coherent backscattering and Anderson localization [37-40], but not nearly to the same extent in the context of angle-independent structural color. Therefore, in our study we aim to show how the physical parameters of the samples - including the particle size and sample thickness - affect the multiple scattering and hence the color. We anticipate that these results will be useful in the development of more precise models of angle-independent structural color and in the formulation of structurally colored materials.

\section{MATERIALS AND METHODS}

\section{A. Synthesis of polystyrene particles}

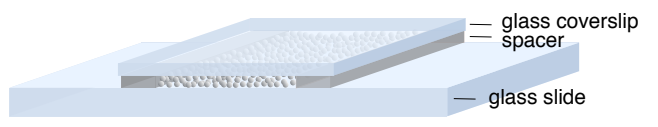

FIG. 2. Diagram of a sample chamber containing a disordered packing of colloidal spheres. The sample chamber consists of a glass slide on the bottom, a glass coverslip on top, and Mylar spacers to set the thickness. It is sealed with UV-curable epoxy.
Our structurally colored materials are made from polystyrene particles. We use emulsion polymerization to synthesize these particles in three sizes: $280 \mathrm{~nm}, 240 \mathrm{~nm}$, and $190 \mathrm{~nm}$ in diameter. All materials are used as received. The polymerization reactor consists of a $500 \mathrm{~mL}$ three-necked round-bottom flask equipped with a reflux condenser, a nitrogen inlet and a mechanical stirrer. In a typical experiment, we dissolve sodium lauryl sulfate (SLS, 99\%, Aldrich) and $3.75 \mathrm{~g}$ of $N$-isopropylacrylamide (NiPAm, 97\%, Aldrich) in $242.5 \mathrm{~mL}$ of deionized (DI) water obtained from a Millipore Milli-Q system in the reactor. We control the diameter of the polystyrene spheres through the amount of SLS. We use $95 \mathrm{mg}$ SLS for the $280 \mathrm{~nm}$ particles, $190 \mathrm{mg}$ for $240 \mathrm{~nm}$, and $285 \mathrm{mg}$ for $190 \mathrm{~nm}$. We add the NiPAm so that we can further functionalize the particles for other experiments not described in this paper [41]. We then add $71.25 \mathrm{~g}$ of styrene $(99 \%$, Aldrich) under vigorous stirring. We heat the mixture to $80^{\circ} \mathrm{C}$ and add $180 \mathrm{mg}$ of potassium persulfate (KPS, $99 \%$, Aldrich) dissolved in $7.5 \mathrm{~mL}$ of DI water. The reaction runs for $8 \mathrm{~h}$. Finally, we wash the resulting particles by dialysis against DI water for $5 \mathrm{~d}$. We measure the particle diameters and polydispersity by image analysis of scanning electron micrographs (SEM). Because the polydispersity index is only $2 \%$, we assume that the particles are monodisperse in most of our scattering calculations (see section IIE).

\section{B. Sample preparation for polarization experiments}

For our polarization experiments, we make disordered, structurally colored films from the polystyrene particles described above (polydispersity 2\%). We start by centrifuging the particles in $25 \mathrm{mM} \mathrm{NaCl}$ for $30 \mathrm{~min}$ at $14000 \mathrm{~g}$ and removing the supernatant. The salt screens the electrostatic interactions between the particles, which is sufficient to prevent them from crystallizing. We then vortex the mixture for $5 \mathrm{~min}$ to resuspend the particles in the remaining liquid. The concentration of the resulting suspension is $45 \% \mathrm{w} / \mathrm{w}$ in water.

We make the films by drying these suspensions in sample chambers of controlled thicknesses, which we make from Mylar spacers sandwiched between glass slides and glass coverslips (Fig. 2). Each chamber is sealed with UV-curable epoxy (Norland Optical Adhesive 68). We pipette a dense suspension into a sample chamber of thickness $77 \mu \mathrm{m}$ and leave a pool of excess suspension at the inlet of the sample chamber. As the water evaporates from the opposite end, more suspension is pulled into the chamber from the pool through capillary action. We periodically replenish the pool as its volume is pulled into the sample chamber. Over the course of $6 \mathrm{~h}$ to $8 \mathrm{~h}$, the particles become densely packed as the water evaporates. We dry the films overnight at room temperature and remove any excess water by drying them in an oven at $60^{\circ} \mathrm{C}$ for several hours. We then seal the sample cham- 
ber with 5-minute epoxy (No. 14250, Devcon). The film of $280 \mathrm{~nm}$ polystyrene particles measured in this paper has an area of $2.8 \mathrm{~cm} \times 1.9 \mathrm{~cm}$ and a thickness of $77 \mu \mathrm{m}$. We estimate the volume fraction from the weight of the polystyrene after drying, the density of the polystyrene, and the volume of the sample chamber.

\section{Polarization measurements}
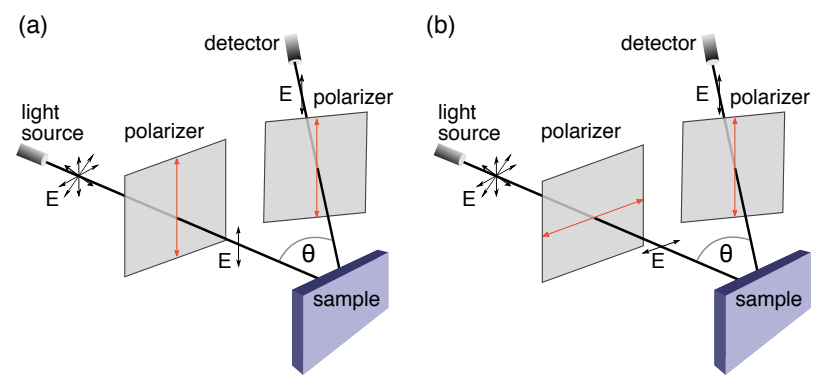

FIG. 3. Setup for polarization measurements. Light from a monochromator shines through a linear polarizer and onto the sample. The light that scatters at an angle $\theta$ to the normal is detected through either (a) a parallel or (b) a perpendicular polarizer. Red arrows indicate the axis of transmission of the polarizer.

We determine the wavelength-dependence of multiple scattering by measuring the spectrum of polarized light. The entire setup is contained inside a spectrophotometer (Agilent Cary 7000 Universal Measurement Spectrophotometer). The sample is mounted on a goniometer (Universal Measurement Accessory of the Agilent Cary 7000 Universal Measurement Spectrophotometer). We set the detection angle $\theta$ to $16^{\circ}$, the smallest angle for which the detector does not cross the incident beam. We choose the largest available aperture, which subtends an angle of $12^{\circ}$, defined by the two edges of the detector and the center of the setup. We also performed measurements with detection angles $\theta=22^{\circ}-76^{\circ}$ to characterize the angle-dependence of our samples (see Fig. 10).

We illuminate the sample with light from a monochromatic source (double out-of-plane Littrow monochromator) sent through a linear polarizer, as shown in Fig. 3. The illuminated spot on the sample is a $5 \mathrm{~mm} \times 5 \mathrm{~mm}$ square. We detect the scattered light through a second polarizer placed in front of the detector (R928 Hamamatsu photomultiplier tube). We measure at wavelength intervals of $1 \mathrm{~nm}$ for $0.3 \mathrm{~s}$ at each wavelength. To measure the diffuse reflectance, we use the same source and spectrophotometer (Fig. 1), but we remove the polarizers and use an integrating sphere accessory instead of the Universal Measurement Accessory. In the integrating sphere measurements, the illuminated spot on the sample is $1 \mathrm{~mm} \times 3 \mathrm{~mm}$, and we measure at wavelength intervals of $1 \mathrm{~nm}$ for $0.1 \mathrm{~s}$ at each wavelength.
We measure both the co-polarized spectrum, which includes any singly scattered light as well as any multiply scattered light that returns to its initial polarization, and the cross-polarized spectrum, which includes only multiply scattered light. In our measurements, light is incident on the glass slide of the sample chamber. We normalize these spectra to correct for the reflection from the glass slide and for the wavelength-dependence of the incident beam and the polarizers:

$$
R_{\mathrm{co}}=\frac{I_{\text {sample }, \mathrm{co}}-I_{\text {glass }, \mathrm{co}}}{I_{\mathrm{inc}} T_{1 \mathrm{v}} T_{2}}
$$

and

$$
R_{\mathrm{cr}}=\frac{I_{\text {sample,cr }}-I_{\text {glass }, \mathrm{cr}}}{I_{\mathrm{inc}} T_{1 \mathrm{~h}} T_{2}},
$$

where $I_{\text {sample, co/cr }}$ is the intensity of light scattered from the sample in the co/cross-polarized setup, $I_{\text {glass, co/cr }}$ is the intensity of light scattered from a glass slide in the co/cross-polarized setup, and $I_{\mathrm{inc}}$ is the source intensity. $T_{1 \mathrm{v} / 1 \mathrm{~h}}$ and $T_{2}$ are the measured transmittances of the two polarizers:

$$
\begin{aligned}
& T_{1 \mathrm{v}}=\frac{I_{1 \mathrm{v}, \text { out }}}{I_{\text {inc }}} \\
& T_{1 \mathrm{~h}}=\frac{I_{1 \mathrm{~h}, \text { out }}}{I_{\text {inc }}} \\
& T_{2}=\frac{I_{2 \mathrm{v}, \text { out }}}{I_{1 \mathrm{v}, \text { out }}},
\end{aligned}
$$

where $I_{1 \mathrm{v} \text {,out }}$ is the intensity of light measured through the first polarizer in the co-polarized setup and $I_{1 \mathrm{~h} \text {,out }}$ is the intensity of light measured through the first polarizer in the cross-polarized setup. $I_{2 \mathrm{v} \text {,out }}$ is the intensity measured through two polarizers oriented vertically. To account for sample inhomogeneity, we report the mean of the reflectance of five separate spots on the sample.

We quantify the amount of multiple scattering through the depolarization ratio [6]

$$
D(\lambda)=\frac{R_{\mathrm{cr}}(\lambda)}{R_{\mathrm{co}}(\lambda)},
$$

where $R$ is reflectance. Pure, high-order multiple scattering should lead to a depolarization of unity, while pure single scattering should lead to a depolarization of zero, because all of the light retains its initial polarization.

We can estimate the amount of single and multiple scattering in the sample by assuming that any light scattered more than once is randomly polarized. Because the polarization of low-order multiple scattering may not be completely randomized, this is a coarse approximation, but it provides a useful estimate. Under this approximation, half of the multiply scattered light is detected through crossed polarizers and the other half is detected through parallel polarizers, while all of the singly scattered light is detected through parallel polarizers. Our estimate of the multiply scattered signal is therefore

$$
R_{\text {multiple scat }}=2 R_{\mathrm{cr}} \text {, }
$$


and our estimate of the singly scattered signal is

$$
R_{\text {single scat }}=R_{\mathrm{co}}-R_{\mathrm{cr}} \text {. }
$$

\section{Sample preparation and measurements of thickness-controlled films}

To determine how the structural color varies with sample thickness, we build polydimethylsiloxane (PDMS) microevaporators with controlled thickness, following the microfluidic protocol in Ref. 42. We make microevaporator channels that are $70 \mu \mathrm{m}$ wide and have thicknesses of $7 \mu \mathrm{m}, 19 \mu \mathrm{m}, 33 \mu \mathrm{m}$ and $47 \mu \mathrm{m}$ (Fig. 4). We then inject a binary suspension of polystyrene particles (by volume, 2 parts of 240-nm-diameter and 1 part of 190-nm-diameter, each at $0.5 \% \mathrm{v} / \mathrm{v}$ ) and $50 \mathrm{mM}$ of sodium chloride into the channels, which have an inlet port but no outlet. As the water evaporates through a 15-um layer of PDMS on the bottom of the channel, the particles pack into a film. Because the evaporation of water is slow $(1 \mathrm{~d}$ to $3 \mathrm{~d}$ at room temperature), adding salt is not sufficient to prevent crystallization. Therefore we use binary suspensions in addition to salt. From the particle size and the location of the main color peak, we estimate the resulting volume fraction as 0.5 , using our single-scattering model. We use this approach instead of the gravimetric estimate we use for the polarization experiments because the microevaporated films do not pack densely along the entirety of their $15-\mathrm{mm}$ length. We measure reflection spectra only in the densely packed regions.

We measure the reflectance spectra of the films with a fiber-optic spectrometer (Ocean Optics HR2000+) attached to an optical microscope (Nikon Eclipse LV-100). We illuminate the films with collimated white light from a halogen lamp, and we collect the scattered light with a $50 \times$ objective (Nikon LU Plan Fluor, NA $=0.8$ ). We normalize the reflection data against the reflection spectrum of an aluminum mirror. (a)

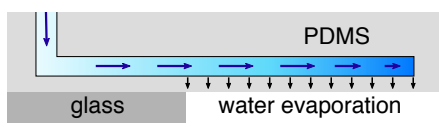

(b)

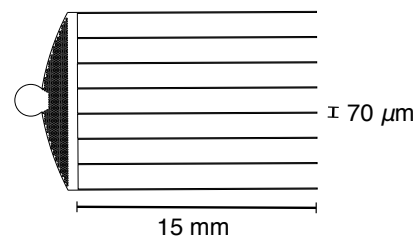

(c)

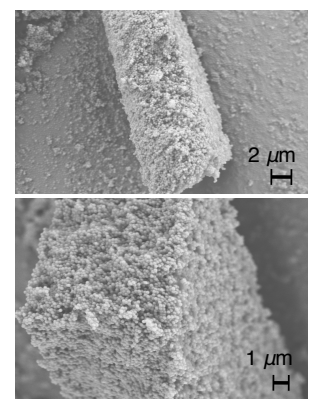

FIG. 4. (a) Microevaporator design [42]. Blue arrows indicate direction of particle flow, and blue color indicates particle packing density. (b) Mask design for the microevaporators. (c) SEM micrographs of assembled structural color films.

To quantify the differences in color between the films, we calculate the color saturation using the CIELUV co- ordinates, which form a perceptual color space:

$$
s_{u v}=\frac{C_{u v}^{*}}{L^{*}}=\frac{\sqrt{\left(u^{*}\right)^{2}+\left(v^{*}\right)^{2}}}{L^{*}},
$$

where $C_{u v}^{*}$ is the chroma, $L^{*}$ corresponds to lightness, and $u^{*}$ and $v^{*}$ correspond to chromaticity [28]. To obtain the $\left(L^{*}, u^{*}, v^{*}\right)$ values, we first calculate the $(X$, $Y, Z)$ color values by integrating the intensity spectrum from the reflectance data multiplied by matching functions that account for the average chromatic response of the human eye [43-45]. Then we calculate the $\left(L^{*}, u^{*}\right.$, $\left.v^{*}\right)$ values using the following transformation:

$$
\begin{aligned}
L^{*} & = \begin{cases}\left(\frac{29}{3}\right)^{3} Y / Y_{n}, & Y / Y_{n} \leq\left(\frac{6}{29}\right)^{3} \\
116\left(Y / Y_{n}\right)^{1 / 3}-16, & Y / Y_{n}>\left(\frac{6}{29}\right)^{3}\end{cases} \\
u^{*} & =13 L^{*}\left(u^{\prime}-u_{n}^{\prime}\right) \\
v^{*} & =13 L^{*}\left(v^{\prime}-v_{n}^{\prime}\right),
\end{aligned}
$$

where $u^{\prime}$ and $v^{\prime}$ are calculated from the $(\mathrm{X}, \mathrm{Y}, \mathrm{Z})$ color values:

$$
\begin{aligned}
u^{\prime} & =\frac{4 X}{X+15 Y+3 Z} \\
v^{\prime} & =\frac{9 Y}{X+15 Y+3 Z} .
\end{aligned}
$$

and where $u_{n}^{\prime}$ and $v_{n}^{\prime}$ are calculated using the above equations, with the $(X, Y, Z)$ color values of a perfect diffuse reflector, $\left(X_{n}, Y_{n}, Z_{n}\right)$, as defined for the CIE Standard Illuminant D65. We use the software package ColorPy [46] to perform this calculation.

\section{E. Single-scattering model calculations}

For our calculations, we use the single-scattering model of Magkiriadou and colleagues [20], but we use the Bruggeman formula for the effective refractive index of the sample [47-49] instead of the Maxwell-Garnett approximation. The Bruggeman formula is symmetric and should therefore be more reliable than Maxwell-Garnett at volume fractions near 0.5 [49], like those in our samples. We account for dispersion in the materials by using the Sellmeier dispersion formula for polystyrene, with parameters that are fit to experimental data [50]. Thus, the effective index also varies with wavelength, as we report in the figure captions describing our measurements.

As discussed in Ref. 20, the model accounts not only for interference between waves scattered from different particles, but also for interference effects within the particles, which lead to backscattering resonances at certain wavelengths. We do, however, neglect near-field effects that might occur in dense packings [39, 40, 51]. Such effects become important when the transport length (see Section III) is comparable to the wavelength. We estimate the transport length from the energy-density coherentpotential approximation (ECPA) [39], a more sophisticated approximation that accounts for near-field effects. 
According to Fig. 4 of Ref. 39, for our disordered samples, which have a volume fraction of 0.5 and particle-radiusto-wavelength ratios that are less than 0.4 , the transport length calculated by ECPA at resonance is eight times the wavelength, and much larger off resonance. Thus, for the small particle sizes (relative to the wavelength) that we use in our samples, it is reasonable to neglect the near-field effects over most of the wavelengths in our measurements. Our approximation is further justified by the good agreement between model and experiment for the position of the main color peak (Fig. 5).

We also modify the model to account for the two particle sizes used in our microevaporator films. Following Scheffold and Mason [52], we write the scattered intensity $I$ as

$$
I \propto \overline{F_{M}(q)} S_{M}(q)
$$

where $\overline{F_{M}(q)}$ is the polydisperse form factor (see below) and $S_{M}(q)$ is the polydisperse structure factor derived by Ginoza and Yasutomi [53]. This structure factor assumes a Schulz size distribution, which tends to a Gaussian distribution when the polydispersity is small. Scheffold and Mason found good agreement between this structure factor and experimental measurements. We calculate the polydisperse form factor $\overline{F_{M}(q)}$ from a size-average:

$$
\overline{F_{M}(q)}=\int_{0}^{\infty} f(\sigma) F(q \sigma) d \sigma,
$$

where $f(\sigma)$ is the Schulz distribution,

$$
f(\sigma)=\left(\frac{t+1}{\sigma_{0}}\right)^{t+1} \frac{\sigma^{t}}{t !} \exp \left(-\sigma \frac{t+1}{\sigma_{0}}\right),
$$

$F(q \sigma)$ is the monodisperse form factor from Mie theory, $\sigma$ the particle diameter, and $\sigma_{0}$ the mean of the size distribution. The parameter $t=\left(1-p^{2}\right) / p^{2}$, where $p$ is the polydispersity index, which accounts for the width of the distribution. For the binary samples used in the microevaporator measurements, we calculate the binary polydisperse form factor as the weighted average of the individual polydisperse form factors.

\section{RESULTS AND DISCUSSION}

We observe three distinct features in the reflectance spectrum of a 77- $\mu \mathrm{m}$ film made from a disordered packing of 280-nm polystyrene spheres: a primary peak near $660 \mathrm{~nm}$, a secondary peak near $580 \mathrm{~nm}$, and an increase in reflectance with decreasing wavelength (Fig. 5a). We examine this sample in detail because its spectral features are well separated by wavelength, which allows us to study each feature independently. The secondary peak is visible in measurements over a narrow range of detection angles (as in Fig. 5a) but not in a measurement using an integrating sphere, where the peaks are broadened and merged (as in Fig. 1b).
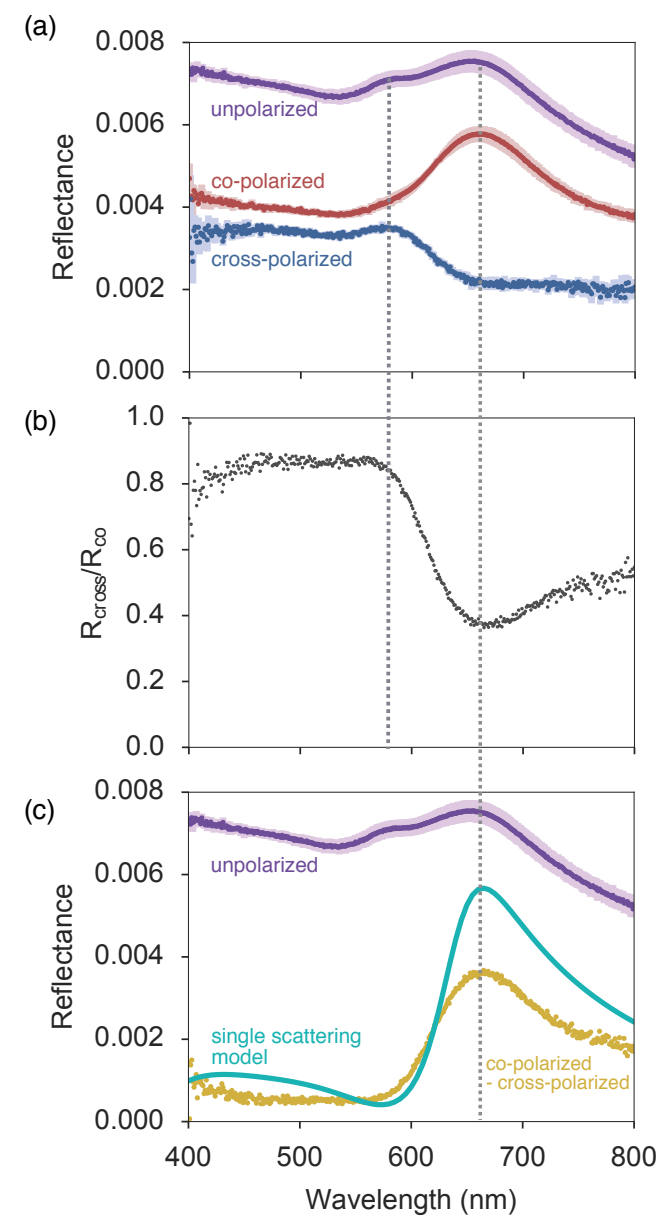

FIG. 5. (a) Unpolarized (purple), co-polarized (red), and cross-polarized (blue) reflectance spectra of a disordered packing of 280-nm polystyrene spheres. Error bars are shown by the shaded regions around each measured spectrum and are twice the standard deviation of five measurements from different areas of the sample film. (b) Depolarization ratio, calculated from Eq. (4). (c) Single-scattering reflectance spectrum (yellow) extracted from data using Eq. (6), compared to spectrum (cyan) calculated from our single-scattering model (Section IIE). The dashed lines indicate the locations of the primary $(660 \mathrm{~nm})$ and secondary $(580 \mathrm{~nm})$ peaks. The calculation uses the following parameters: a volume fraction of 0.53 , corresponding to the measured volume fraction, a diameter of $280 \mathrm{~nm}$, and an effective refractive index ranging from 1.322 at $400 \mathrm{~nm}$ to 1.296 at $800 \mathrm{~nm}$. See Fig. 10 for the angle-dependence of the co- and cross- polarized spectra.

Both the polarization experiments and the singlescattering model suggest that the measured primary peak near $660 \mathrm{~nm}$ comes from single scattering: the depolarization ratio is minimized at the peak wavelength (Fig. 5b), and the model predicts a peak at the same wavelength. The absence of this peak in the cross-polarized spectrum suggests that multiple scattering does not contribute to the peak. The agreement with the single-scattering model suggests that the primary peak is due to the interference between waves scattered from the structure. 
Indeed, we find that the predictions of the singlescattering model agree well with the single-scattering spectrum extracted from the data through Eq. (6), as shown in Fig. 5c. The subtraction of the cross-polarized spectrum removes much of the low-wavelength intensity and narrows the primary peak, resulting in a spectrum that more closely matches that predicted by the singlescattering model. This agreement tells us that the singlescattering model not only predicts the primary peak of the reflectance, but also gives a reasonable estimate for how much single scattering contributes to the reflectance.

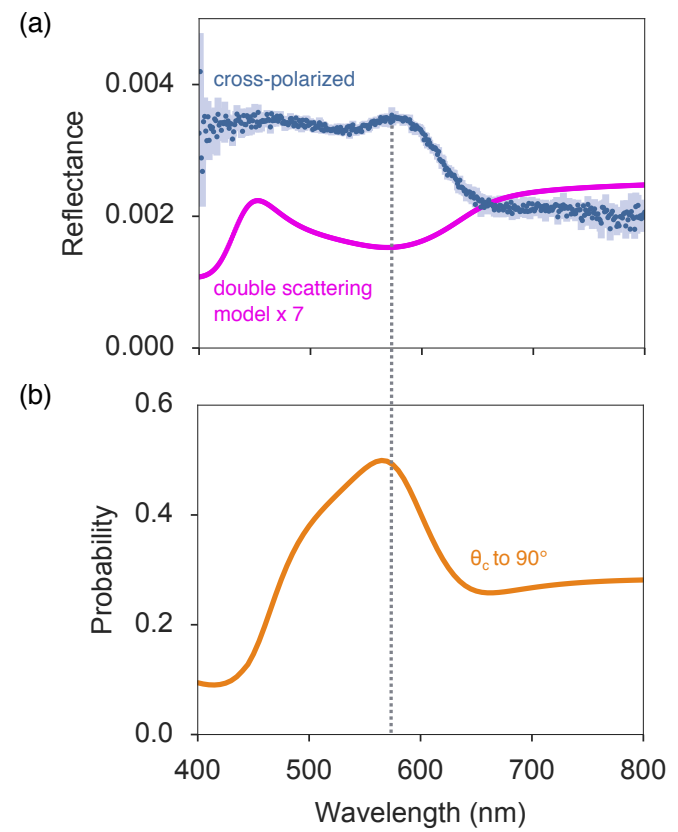

FIG. 6. Total internal reflection, rather than double scattering, explains the peak in the cross-polarized spectrum. (a) Cross-polarized (blue) spectrum from Fig. 5a and reflectance calculated with a double-scattering model (magenta) of a disordered packing of $280-\mathrm{nm}$ polystyrene spheres, assuming a volume fraction of 0.53 and detection angles ranging from $\theta=10^{\circ}$ to $22^{\circ}$ (see Fig. 3). The effective refractive index, calculated using the Bruggeman approximation, ranges from 1.322 at $400 \mathrm{~nm}$ to 1.296 at $800 \mathrm{~nm}$. The double-scattering reflectance is multiplied by a factor of 7 for clarity. Error bars for the cross-polarized spectrum are shown in light blue and are twice the standard deviation of 5 measurements from different areas of the sample film. (b) Probability of single scattering into the totally internally reflected angular range. The critical angle $\theta_{c}$ for this sample, as calculated from the wavelength-dependent effective refractive index, ranges from $49^{\circ}$ at $400 \mathrm{~nm}$ to $50^{\circ}$ at $800 \mathrm{~nm}$,

Having explained the origin of the primary peak in terms of single scattering, we now turn to the spectral features that deviate from the predictions of the model: the secondary peak and the increase in reflectance toward low wavelengths. The secondary peak in the unpolarized spectrum comes from the peak in the cross-polarized reflectance, which is due to multiple scattering. Following the example of Noh and coworkers [6], we first examine whether double scattering can explain this peak.

In our double-scattering model, the reflectance is proportional to the integral of the phase function of two consecutive scattering events. The phase function is the probability that light is scattered in a certain direction $\theta^{\prime}$ :

$$
p\left(\theta^{\prime}\right)=\frac{1}{\sigma_{\text {scat }}} \frac{\mathrm{d} \sigma_{\text {scat }}}{\mathrm{d} \Omega}\left(\theta^{\prime}\right),
$$

where the scattering angle $\theta^{\prime}=180^{\circ}-\theta, \mathrm{d} \sigma_{\text {scat }} / \mathrm{d} \Omega$ is the differential scattering cross-section, and $\sigma_{\text {scat }}$ is the total scattering cross-section [54]. We assume that in our disordered samples, the phase function is isotropic in the azimuthal angle $\phi$. The double-scattering phase function is

$$
p_{\text {double }}=p_{\text {first }} p_{\text {second }},
$$

where $p_{\text {first }}$ and $p_{\text {second }}$ are the phase functions of the first and second scattering events. Both are calculated from the single-scattering model, $p_{\text {second }}$ by rotating $p_{\text {first }}$ from the lab frame to the scattering plane of the second event.

Double scattering does not explain the secondary peak in our samples, as shown in Fig. 6a. Noh and colleagues performed double-scattering calculations based on smallangle X-ray scattering data and found that double scattering does appear to explain the secondary peak in the reflectance of cotinga feathers [6]. Our results may differ from theirs because of differences in structure: our structures consist of polystyrene spheres in an air matrix, whereas bird feathers are "inverse" structures of air spheres in a keratin matrix.

In our samples, the secondary peak appears to be due to scattering from totally internally reflected waves. When light is scattered toward the sample interface, some fraction is totally internally reflected back into the sample, where it can scatter again. To contribute to the reflectance, the totally internally reflected light must scatter at least once more before it exits the sample. By integrating the phase function for a single-scattering event over the angular range for total internal reflection, we find the probability that singly scattered light is totally internally reflected. In this calculation, we consider the interface to be between sample and air, since the refraction due to the glass slide cancels when we apply Snell's law to both the sample-glass and glass-air boundaries.

The probability is peaked at a wavelength that matches that of the observed secondary peak (Fig. 6b). Although the probability does not quantitatively predict the contribution of totally internally reflected light to the reflection spectrum - a more sophisticated model is needed for such a prediction - the agreement between the peak positions strongly suggests that the secondary peak is due to totally internally reflected light. The single-scattering model does not capture this effect, because the model assumes that totally internally reflected light is lost. We note that this effect might also be present in the bird feathers examined by Noh and colleagues. 
The effect of internal reflection on multiply scattering media has been investigated by $\mathrm{Zhu}$ and colleagues [55], but their model assumes that light from inside the sample impinges on the boundary over a uniform distribution of angles, which is not the case for the single and low-order multiple scattering in our samples. The angledependence of singly scattered light in our samples comes from the angle-dependence of the structure factor and the form factor, which are used to calculate the differential scattering cross-section.

(a)

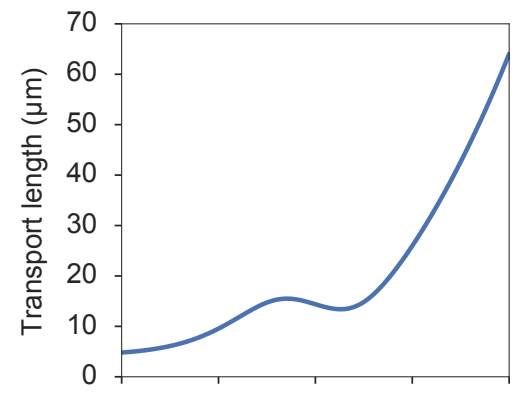

(b)

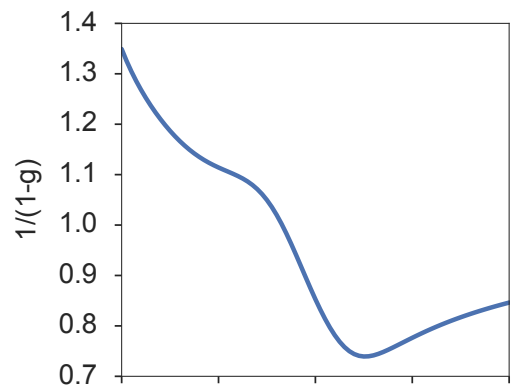

(c)

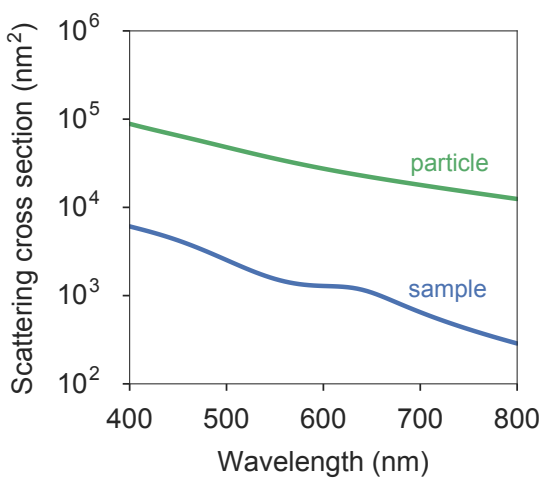

FIG. 7. (a) Transport length, (b) asymmetry parameter factor, and (c) scattering cross-sections calculated for a sample with $280-\mathrm{nm}$ polystyrene spheres at a volume fraction of 0.53 and an effective refractive index ranging from 1.322 at $400 \mathrm{~nm}$ to 1.296 at $800 \mathrm{~nm}$. The particle cross-section is calculated with Mie theory, and the sample cross-section is calculated with the single-scattering model. Note that the cross section is on a $\log$ scale.

To understand the origin of the large depolarization at low wavelengths and the accompanying rise in scattering toward the blue, we return to the single-scattering model. Although this model cannot capture the contribu- tion of multiple scattering to the spectrum, it can predict the propensity for multiple scattering. This propensity is characterized by the transport length $l^{*}(\lambda)$, which is the distance that light propagates into the sample before its direction is randomized [56-58]. The shorter the transport length at a fixed film thickness, the higher the propensity for multiple scattering.

The transport length $l^{*}$ is related to the asymmetry parameter $g$ and scattering length $l_{\text {scat }}$ :

$$
l^{*}=\frac{l_{\mathrm{scat}}}{1-g},
$$

where $g=\langle\cos (\theta)\rangle$ and $\theta$ is the scattering angle. The scattering length $l_{\text {scat }}$ is the average distance between scattering events and is calculated as $1 /\left(N \sigma_{\text {scat }}\right)$, where $N$ is the number density and $\sigma_{\text {scat }}$ is the scattering crosssection of the sample [59]. We can then express the transport length $l^{*}$ as

$$
l^{*}=\frac{1}{N \sigma_{\text {scat }}(1-g)} .
$$

We use our single-scattering model to calculate the sample scattering cross-section and the asymmetry parameter, which includes the contributions of both the form factor and the structure factor within our effective-medium approximation [20].

Our calculations show that the transport length has a local minimum at approximately the wavelength of the primary reflectance peak (Fig. 7a). This minimum is not surprising, since constructive interference contributes to strong backscattering at the structural resonance [57], leading to both a minimum in the asymmetry parameter factor $1 /(1-g)$ (see Fig. 7b) and a local maximum in the sample scattering cross-section (see Fig. 7c). Although a minimum in the transport length should correspond to a higher propensity for multiple scattering, the crosspolarized spectrum does not show a peak at the same wavelength, indicating that high-order multiple scattering does not contribute significantly to the main peak.

More interestingly, the transport length for this sample is smallest (less than $10 \mu \mathrm{m}$ ) at wavelengths from $400 \mathrm{~nm}$ to $500 \mathrm{~nm}$, as shown in Fig. 7a. This feature does not appear to be due to the asymmetry parameter factor, $1 /(1-g)$, which increases with decreasing wavelengths. If this factor were the only contribution to the transport length, the transport length would also increase with decreasing wavelength, in contradiction with our results.

Instead, the decrease in transport length at short wavelengths appears to be due to the scattering from individual particles. We find that the single-particle scattering cross-section, calculated with Mie theory, increases by a factor of 7 from $800 \mathrm{~nm}$ to $400 \mathrm{~nm}$, leading to an increase in the sample cross-section by more than an order of magnitude at short wavelengths (Fig. 7c).

The transport length calculations allow us to explain how the structural color changes with the thickness of the sample, as illustrated in Fig. 8. As the sample thickness 

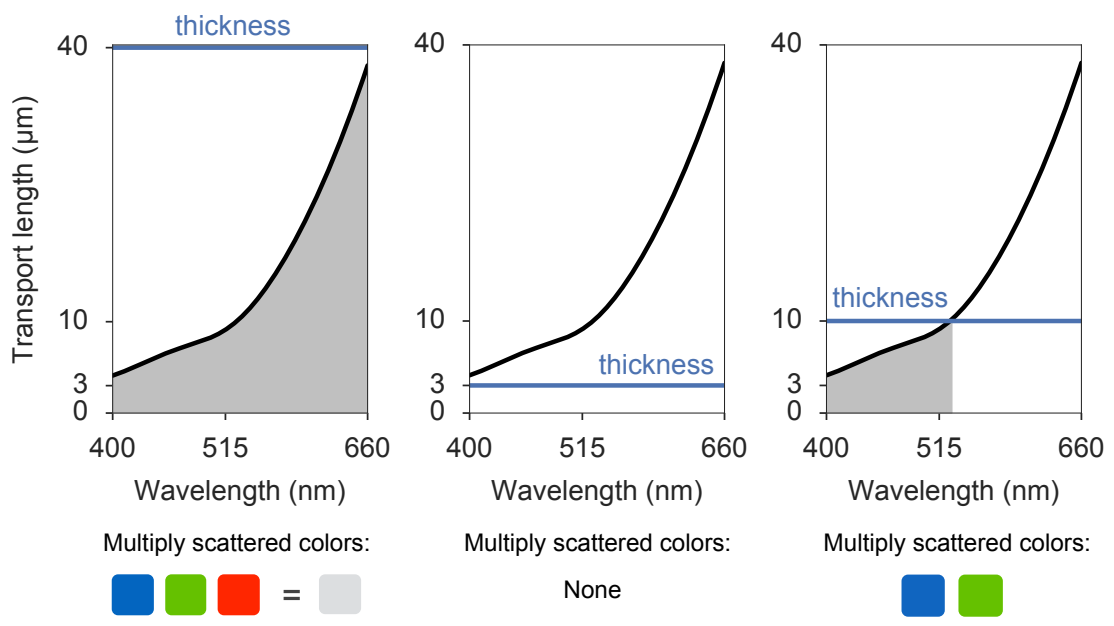

FIG. 8. Transport length calculations for a disordered packing of a binary mixture of polystyrene particles (2 parts by volume of 240-nm-diameter and 1 part of 190 -nm-diameter, each with a polydispersity of $2 \%$ ) in air assuming a volume fraction of 0.5 and an effective refractive index ranging from 1.302 at $400 \mathrm{~nm}$ to 1.285 at $660 \mathrm{~nm}$. The panels show schematically how changing the sample thickness relative to the transport length changes how certain colors are reflected from the sample.

changes in comparison to the transport length, different colors can appear in the reflectance spectra. When the thickness is larger than the transport length in the entire spectrum, all wavelengths are likely to be multiply scattered, and the resulting color is white. When the thickness is smaller than the transport length at any wavelength, light is likely to pass through the film without scattering, and the sample becomes transparent. When the thickness is approximately the transport length at the reflectance peak, the resulting structural color is saturated, meaning that, on average, the scattering at wavelengths close to the reflectance peak is high relative to that at other wavelengths. Although the diagram in Fig. 8 does not allow one to quantitatively predict the color, it illustrates the important physical considerations needed to design saturated colors.

To validate this physical picture, we assemble four structurally colored films of different thicknesses inside microevaporators and measure the reflectance spectrum at each thickness. We use the saturation of the spectrum as a measure of the amount of multiple scattering in each sample. The highest color saturations occur at thicknesses of $19 \mu \mathrm{m}$ and $33 \mu \mathrm{m}$ (Fig. 9), which are 2-3 times larger than the transport length $(10 \mu \mathrm{m}$; see Fig. 8) calculated at the primary peak. Samples smaller than this transport length are translucent, as shown by the low reflectance of the 7-um film. In fact, in the image of this film, the dark background is visible through the sample. When the sample is thicker than the transport length at all wavelengths, the color is desaturated, as shown by the spectrum and image of the $47-\mu \mathrm{m}$ film. These results agree qualitatively with the predictions shown in Fig. 8.

In addition, the main peaks in the more saturated samples are narrower and slightly shifted with respect to the peaks in the thickest and thinnest films. In the thickest film, scattering at wavelengths off resonance shifts the

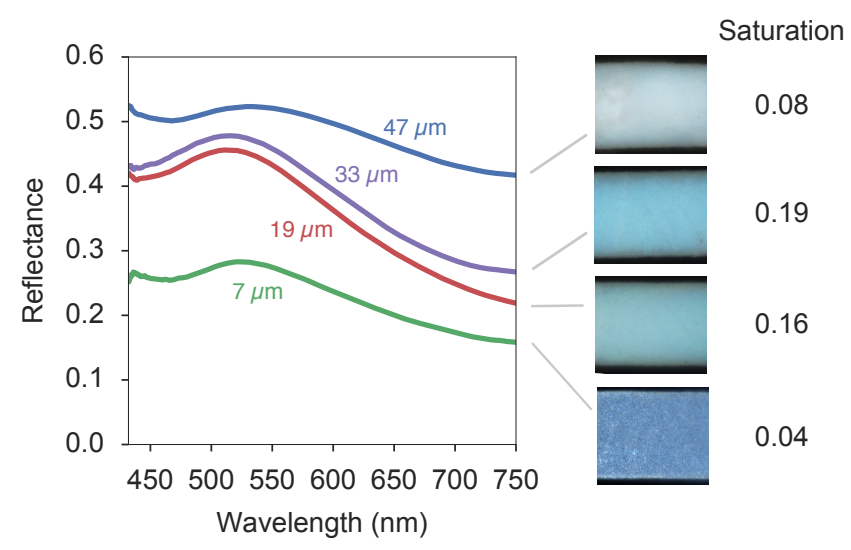

FIG. 9. Reflectances of films made in microevaporators of different thicknesses, as indicated by the labels near each curve. The films consist of packings of polystyrene nanoparticles (2 parts of 240-nm-diameter and 1 part of 190-nm-diameter by volume, each with a polydispersity of $2 \%$ ). At right, darkfield optical micrographs show the color at each thickness. The color saturations are listed to the right of each image.

location of the peak. In the thinnest film, the peak is flattened because the sample has less scattering overall. Also, this film shows a much lower reflection at short wavelengths, consistent with our analysis in Figure 7. The changes in the shapes of the peaks with changing thickness illustrate the central role that light transport plays in setting the color.

We conclude that although the single-scattering model cannot quantitatively predict the reflectance spectrum of samples with large thicknesses, it can predict how the multiple scattering (and color saturation) varies with wavelength and sample thickness. This prediction comes from calculating the transport length as a function of 
wavelength and comparing it to the sample thickness. Though the transport length is commonly used to understand multiple scattering in contexts such as weak localization [37, 39, 40], our results show that it is also useful for understanding how multiple scattering compromises color saturation - for example, through the rise in reflectance at short wavelengths.

The model can be used to design saturated colors in the absence of absorption: after calculating the transport length as a function of wavelength, one can make a sample with saturated color by choosing a film thickness that is on the order of (more precisely, 2 to 3 times) the transport length at the reflection peak. In the absence of absorption, this design rule is quantitative, in that the transport length calculated from the single-scattering model provides a quantitative estimate for the sample thickness required for optimal saturation. The estimate is coarse but provides a useful starting point for sample design.

In the presence of broadband absorbers, which are often used to tune saturation $[15,28]$, the transport length is still an important parameter. But now a third lengthscale must be taken into account: the absorption length, which is set by the concentration of absorbing material, among other factors. We consider several possible orderings of these three length scales, assuming, for simplicity, that the absorption length does not vary significantly with wavelength. Let $l_{\text {abs }}$ be the absorption length, $l^{*}$ be the transport length at the reflection peak, and $L$ be the sample thickness. When the thickness is the largest of the three lengthscales, optimal saturation should correspond to an absorption length that is comparable to the transport length: $l_{\text {abs }} \sim l^{*} \ll L$. If the absorption length were much smaller than the transport length $\left(l_{\text {abs }} \ll l^{*} \ll L\right)$, the scattering would be weak and absorption would dominate. If the absorption length were much larger than the transport length $\left(l^{*} \ll l_{\text {abs }} \ll L\right)$, multiple scattering would not be suppressed. If the absorption length is the largest of the three lengthscales, we obtain the design rule specified above: $l^{*} \sim L \ll l_{\text {abs }}$. Finally, when the transport length is the largest of the three lengthscales $\left(l_{\text {abs }}, L \ll l^{*}\right)$, we expect only weak color. The sample will be transparent for $L \ll l_{\text {abs }} \ll l^{*}$ and black for $l_{\text {abs }} \ll L \ll l^{*}$.

\section{CONCLUSIONS}

We have shown that three spectral features determine the structural color of disordered colloidal materials, and we have established their origins. The location of the main peak in the reflectance spectrum can be predicted accurately from a single-scattering model that accounts for the effective index of the material and its glassy structure, as shown previously [20]. Our measurements show that near the peak, most of the light is singly scattered. However, the peak is broader than predicted by the model because of a peak at a slightly smaller wavelength that arises from multiple scattering and total internal reflection. The third spectral feature, an increase in scattering toward shorter wavelengths, leads to the largest deviation from the model predictions. We have shown that this multiple scattering is due to the scattering from individual particles, and its increase is related to the increase in the single-particle scattering cross-section.

We have also shown that the single-scattering model is a useful tool for understanding and predicting structural color. The model reproduces the peak and the shape of the measured single-scattering spectrum. Even though it does not account for the contribution of multiple scattering to the reflectance spectra, it can be used to calculate the transport length, which in turn can be used to predict the onset of multiple scattering.

To make samples with saturated structural color for applications, it is necessary to have high scattering at the primary peak and minimal scattering off peak. The saturation can be maximized by varying the sample thickness and/or by adding broadband absorbers such as carbon black to the material [15]. In many applications, however, it may not be possible to add absorbers. For example, in reflective displays, absorbing materials lead to heating under illumination. As shown here, the single-scattering model provides a way to predict the optimal thickness, in the absence of absorption, based on the wavelength dependence of the transport length. Even in the presence of absorption, an understanding of the physical origins of multiple scattering is important, since multiple scattering increases the path length of light and thus the probability of being absorbed [60]. Thus, any subsequent models that attempt to predict how a given amount of broadband absorber affects the color must account for multiple scattering as well. We leave the development of a model that can predict the reflection spectrum in the presence of both multiple scattering and absorption for future work.

\section{ACKNOWLEDGMENTS}

Anna B. Stephenson acknowledges the support of the National Science Foundation (NSF) Graduate Research Fellowship Program. This work was funded by the Harvard MRSEC under grant number DMR-1420570 and by the Xerox University Affairs Committee. In addition, this work was performed in part at the Center for Nanoscale Systems (CNS), a member of the National Nanotechnology Coordinated Infrastructure Network (NNCI), which is supported by the National Science Foundation under grant number EECS-1541959. CNS is part of Harvard University. We thank Dr. Arthur McClelland for helpful discussions on the polarization setup and Dr. Ming Xiao and Anastasia Ershova for suggestions on our manuscript. 
(a)

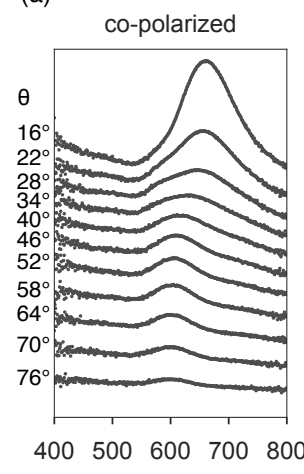

(b)

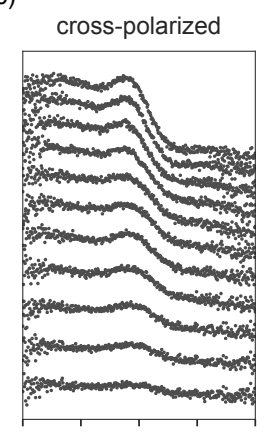

(c) co - cross-polarized (d)

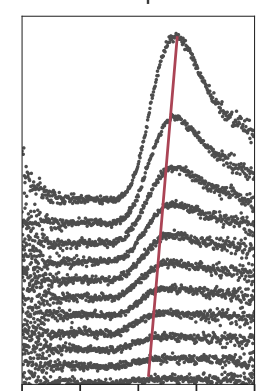

(d) single scattering model

Wavelength $(\mathrm{nm})$

FIG. 10. Angle-dependence of polarization spectra. (a) Co-polarized reflection spectra. (b) Cross-polarized reflection spectra. (c) Co- minus cross-polarized reflection spectra. (d) Reflection spectra calculated from the single-scattering model. Each curve in the panels corresponds to a different detection angle $\theta$. Spectra are offset along the y-axis for clarity. For the measurements in (a)-(c), the sample and experimental setup are the same as in Fig. 5a. For the calculation in (d), we use a volume fraction of 0.53 , corresponding to the measured volume fraction, a diameter of $280 \mathrm{~nm}$, and an effective refractive index ranging from 1.322 at $400 \mathrm{~nm}$ to 1.296 at $800 \mathrm{~nm}$.

\section{APPENDIX: ANGLE-DEPENDENCE OF POLARIZATION SPECTRA}

Measurements of the angle-dependence of the polarization spectra reveal that the sample is disordered. The primary peak blueshifts as the detection angle $\theta$ increases (Fig. 10a, c). This blueshift matches the peak shift predicted for a disordered colloidal sample, as calculated from the single-scattering model.

[1] J. D. Joannopoulos, S. G. Johnson, J. N. Winn, and R. D. Meade, Photonic Crystals: Molding the Flow of Light, 2nd ed. (Princeton University Press, Princeton, 2008).

[2] R. O. Prum, R. H. Torres, S. Williamson, and J. Dyck, Nature 396, 28 (1998).

[3] M. D. Shawkey, V. Saranathan, H. Palsdottir, J. Crum, M. H. Ellisman, M. Auer, and R. O. Prum, Journal of The Royal Society Interface 6, S213 (2009).

[4] E. R. Dufresne, H. Noh, V. Saranathan, S. G. J. Mochrie, H. Cao, and R. O. Prum, Soft Matter 5, 1792 (2009).

[5] H. Noh, S. F. Liew, V. Saranathan, S. G. J. Mochrie, R. O. Prum, E. R. Dufresne, and H. Cao, Advanced Materials 22, 2871 (2010).

[6] H. Noh, S. F. Liew, V. Saranathan, R. O. Prum, S. G. J. Mochrie, E. R. Dufresne, and H. Cao, Physical Review E 81, 051923 (2010).

[7] H. Noh, S. F. Liew, V. Saranathan, R. O. Prum, S. G. J. Mochrie, E. R. Dufresne, and H. Cao, Optics Express 18, 11942 (2010).

[8] H. Yin, B. Dong, X. Liu, T. Zhan, L. Shi, J. Zi, and E. Yablonovitch, Proceedings of the National Academy of Sciences 109, 10798 (2012).

[9] V. Saranathan, J. D. Forster, H. Noh, S.-F. Liew, S. G. J. Mochrie, H. Cao, E. R. Dufresne, and R. O. Prum, Journal of The Royal Society Interface 9, 2563 (2012).
[10] P. D. García, R. Sapienza, A. Blanco, and C. López, Advanced Materials 19, 2597 (2007).

[11] Y. Takeoka, M. Honda, T. Seki, M. Ishii, and H. Nakamura, ACS Applied Materials \& Interfaces 1, 982 (2009).

[12] K. Ueno, A. Inaba, Y. Sano, M. Kondoh, and M. Watanabe, Chemical Communications , 3603 (2009).

[13] P. D. García, R. Sapienza, and C. López, Advanced Materials 22, 12 (2010).

[14] M. Harun-Ur-Rashid, A. Bin Imran, T. Seki, M. Ishii, H. Nakamura, and Y. Takeoka, ChemPhysChem 11, 579 (2010).

[15] J. D. Forster, H. Noh, S. F. Liew, V. Saranathan, C. F. Schreck, L. Yang, J. G. Park, R. O. Prum, S. G. J. Mochrie, C. S. O'Hern, H. Cao, and E. R. Dufresne, Advanced Materials 22, 2939 (2010).

[16] I. Lee, D. Kim, J. Kal, H. Baek, D. Kwak, D. Go, E. Kim, C. Kang, J. Chung, Y. Jang, S. Ji, J. Joo, and Y. Kang, Advanced Materials 22, 4973 (2010).

[17] M. Retsch, M. Schmelzeisen, H.-J. Butt, and E. L. Thomas, Nano Letters 11, 1389 (2011).

[18] Y. Takeoka, S. Yoshioka, A. Takano, S. Arai, K. Nueangnoraj, H. Nishihara, M. Teshima, Y. Ohtsuka, and T. Seki, Angewandte Chemie International Edition 125, 7402 (2013).

[19] J.-G. Park, S.-H. Kim, S. Magkiriadou, T. M. Choi, Y.S. Kim, and V. N. Manoharan, Angewandte Chemie 
International Edition 53, 2899 (2014).

[20] S. Magkiriadou, J. G. Park, Y.-S. Kim, and V. N. Manoharan, Physical Review E 90, 062302 (2014).

[21] S. Yoshioka and Y. Takeoka, ChemPhysChem 15, 2209 (2014).

[22] D. Ge, L. Yang, G. Wu, and S. Yang, Journal of Materials Chemistry C 2, 4395 (2014).

[23] Y. Takeoka, Polymer Journal 47, 106 (2014).

[24] M. Teshima, T. Seki, R. Kawano, S. Takeuchi, S. Yoshioka, and Y. Takeoka, Journal of Materials Chemistry C 3, 769 (2015).

[25] F. Wang, X. Zhang, Y. Lin, L. Wang, and J. Zhu, ACS Applied Materials \& Interfaces 8, 5009 (2016).

[26] C.-F. Lai, Y.-C. Wang, and H.-C. Hsu, Journal of Materials Chemistry C 4, 398 (2016).

[27] X. Yang, D. Ge, G. Wu, Z. Liao, and S. Yang, ACS Applied Materials \& Interfaces 8, 16289 (2016).

[28] M. Iwata, M. Teshima, T. Seki, S. Yoshioka, and Y. Takeoka, Advanced Materials 29, 1605050 (2017).

[29] S.-H. Kim, S. Magkiriadou, D. K. Rhee, D. S. Lee, P. J. Yoo, V. N. Manoharan, and G.-R. Yi, ACS Applied Materials \& Interfaces 9, 24155 (2017).

[30] B. Q. Dong, X. H. Liu, T. R. Zhan, L. P. Jiang, H. W. Yin, F. Liu, and J. Zi, Optics Express 18, 14430 (2010).

[31] M.-L. Lo and C.-C. Lee, Applied Optics 53, A399 (2014).

[32] F. Cheng, J. Gao, T. S. Luk, and X. Yang, Scientific Reports 5, 11045 (2015).

[33] H. Galinski, G. Favraud, H. Dong, J. S. T. Gongora, G. Favaro, M. Döbeli, R. Spolenak, A. Fratalocchi, and F. Capasso, Light: Science \& Applications 6, e16233 (2017).

[34] M. Xiao, Z. Hu, Z. Wang, Y. Li, A. D. Tormo, N. L. Thomas, B. Wang, N. C. Gianneschi, M. D. Shawkey, and A. Dhinojwala, Science Advances 3, e1701151 (2017).

[35] C. J. Chandler, B. D. Wilts, J. Brodie, and S. Vignolini, Advanced Optical Materials 5, 1600646 (2017).

[36] S. Cho, T. S. Shim, J. H. Kim, D.-H. Kim, and S.-H. Kim, Advanced Materials 29, 1 (2017).

[37] D. S. Wiersma, M. P. van Albada, and A. Lagendijk, Physical Review Letters 75, 1739 (1995).

[38] L. S. Froufe-Perez, M. Engel, J. J. Sáenz, and F. Scheffold, Proceedings of the National Academy of Sciences 114, 9570 (2017).

[39] G. J. Aubry, L. Schertel, M. Chen, H. Weyer, C. M. Aegerter, S. Polarz, H. Cölfen, and G. Maret, Physical Review A 96, 043871 (2017).

[40] L. Schertel, I. Wimmer, P. Besirske, C. M. Aegerter, G. Maret, S. Polarz, and G. J. Aubry, Physical Review
Materials 3 (2019).

[41] J.-G. Park, W. B. Rogers, S. Magkiriadou, T. Kodger, S.-H. Kim, Y.-S. Kim, and V. N. Manoharan, Optical Materials Express 7, 253 (2017).

[42] A. Merlin, J.-B. Salmon, and J. Leng, Soft Matter 8, 3526 (2012).

[43] J. Schanda, Colorimetry: Understanding the CIE System (Wiley Interscience, 2007).

[44] E. C Carter, Y. Ohno, M. R. Pointer, A. R. Robertson, R. Sève, J. D. Schanda, and K. Witt, CIE 15: Technical Report: Colorimetry, 3rd edition, Tech. Rep. (CIE, Central Bureau, Vienna, 2004).

[45] C. Poynton, Digital Video and HDTV. (MorganKaufmann, 2003).

[46] M. Kness, "ColorPy - A python package for handling physical descriptions of color and light spectra," The source package can be found at https://github.com/ markkness/ColorPy (2008).

[47] D. A. G. Bruggeman, Annalen der Physik 416, 636 (1935).

[48] D. A. G. Bruggeman, Annalen der Physik 417, 645 (1936).

[49] V. A. Markel, Journal of the Optical Society of America A 33, 1244 (2016).

[50] N. Sultanova, S. Kasarova, and I. Nikolov, Acta Physica Polonica A 116, 585 (2009).

[51] R. Rezvani Naraghi, S. Sukhov, J. J. Sáenz, and A. Dogariu, Physical Review Letters 115, 203903 (2015).

[52] F. Scheffold and T. G. Mason, Journal of Physics: Condensed Matter 21, 332102 (2009).

[53] M. Ginoza and M. Yasutomi, Journal of the Physical Society of Japan 68, 2292 (1999).

[54] C. F. Bohren and D. R. Huffman, Absorption and scattering of light by small particles (Wiley-VCH Verlag $\mathrm{GmbH}$ Co. KGaA, 2004).

[55] J. X. Zhu, D. J. Pine, and D. A. Weitz, Physical Review A 44, 3948 (1991).

[56] P. D. Kaplan, A. D. Dinsmore, A. G. Yodh, and D. J. Pine, Physical Review E 50, 4827 (1994).

[57] L. F. Rojas-Ochoa, J. M. Mendez-Alcaraz, J. J. Sáenz, P. Schurtenberger, and F. Scheffold, Physical Review Letters 93 (2004).

[58] M. Reufer, L. F. Rojas-Ochoa, S. Eiden, J. J. Sáenz, and F. Scheffold, Applied Physics Letters 91, 171904 (2007).

[59] A. Ishimaru, Wave propagation and scattering in random media (IEEE Press: New York and Oxford University Press: Oxford, 1991).

[60] D. E. McCoy, T. Feo, T. A. Harvey, and R. O. Prum, Nature Communications 9, 1 (2018). 